\title{
ON THE SEMI-SIMPLICITY OF THE QUANTUM COHOMOLOGY ALGEBRAS OF COMPLETE INTERSECTIONS
}

\author{
Gang Tian and Geng Xu
}

\section{Introduction}

In this short note, we study the semi-simplicity of the quantum cohomology algebras of smooth Fano complete intersections in projective space.

Let $V$ be a smooth Fano manifold with a symplectic form $\omega$. The quantum cohomology on $V$ is the cohomology $H^{*}\left(V, \mathbb{Z}\left\{H_{2}(V)\right\}\right)$ with a ring structure defined by the GW-invariants. The Novikov ring $\mathbb{Z}\left\{H_{2}(V)\right\}$ can be described as follows: choose a basis $q_{1}, \cdots, q_{s}$ of $H_{2}(V, \mathbb{Z})$, we identify the monomial $q^{d}=$ $q_{1}^{d_{1}} \cdots q_{s}^{d_{s}}$ with the sum $\sum_{i=1}^{s} d_{i} q_{i}$. This turns $H_{2}(V)$ into a multiplicative ring, i.e., $q^{d} \cdot q^{d^{\prime}}=q^{d+d^{\prime}}$. This multiplicative ring has a natural grading defined by $\operatorname{deg}\left(q^{d}\right)=2 c_{1}(V)\left(\sum d_{i} q_{i}\right)$. Then $\mathbb{Z}\left\{H_{2}(V)\right\}$ is the graded homogeneous ring generated by all formal power series $\sum_{d=\left(d_{1}, \cdots, d_{s}\right)} n_{d} q^{d}$ satisfying: $n_{d} \in \mathbb{Z}$, all $q^{d}$ with $n_{d} \neq 0$ have the same degree, and the number of $n_{d}$ with $\omega\left(\sum d_{i} q_{i}\right) \leq c$ is finite for any $c>0$.

Now we can define a ring structure on $H^{*}\left(V, \mathbb{Z}\left\{H_{2}(V)\right\}\right)$. For any $\alpha^{*}, \beta^{*}$ in $H^{*}(V, \mathbb{Z})$, we define the quantum multiplication $\alpha^{*} \bullet \beta^{*}$ by

$$
\alpha^{*} \bullet \beta^{*}(\gamma)=\sum_{A \in H_{2}(V, \mathbb{Z})} \Psi_{(A, 0,3)}^{V}(\alpha, \beta, \gamma) q^{A}
$$

where $\gamma \in H_{*}(V, \mathbb{Z})$, and $\Psi_{(A, 0,3)}^{V}(\alpha, \beta, \gamma)$ are the Gromov-Witten invariants (cf. $[\mathrm{RT}])$.

In fact, there is a family of quantum multiplications. Let $\left\{\beta_{a}\right\}_{1 \leq a \leq L}$ be an integral basis of $H_{*}(V, \mathbb{Z})$ modulo torsions. Any $w \in H^{*}(V, \mathbb{C})$ can be written as $\sum t_{a} \beta_{a}^{*}$. Clearly, $w \in H^{*}(V, \mathbb{Z})$ if all $t_{a}$ are integers. We define the quantum multiplication $\bullet$ by

$$
\alpha^{*} \bullet_{w} \beta^{*}(\gamma)=\sum_{A} \sum_{k \geq 0} \frac{\epsilon\left(\left\{a_{i}\right\}\right)}{k !} \Psi_{(A, 0, k+3)}^{V}\left(\alpha, \beta, \gamma, \beta_{a_{1}}, \cdots, \beta_{a_{k}}\right) t_{a_{1}} \cdots t_{a_{k}} q^{A}
$$

Received March 27, 1997.

The first author was partially supported by a NSF grant.

The second author was partially supported by a NSF grant. 
where $\alpha, \beta, \gamma \in H_{*}(V, \mathbb{Z}), \epsilon\left(\left\{a_{i}\right\}\right)$ is the sign of the induced permutation on odd dimensional $\beta_{a}$, and $\Psi_{(A, 0, k+3)}^{V}\left(\alpha, \beta, \gamma, \beta_{a_{1}}, \cdots, \beta_{a_{k}}\right)$ are the Gromov-Witten invariants. Obviously, this multiplication reduces to $\bullet$ at $w=0$. It was shown in $[\mathrm{RT}]$ that the quantum multiplications $\bullet_{w}$ are associative.

Now we restrict ourselves to the case $w \in W=H^{\text {even }}(V, \mathbb{C})$. In this case, Dubrovin $[\mathrm{D}]$ observed that the quantum multiplications $\bullet_{w}$ induce the structure of a Frobenius algebra on $W$. For any $w \in W, w=\sum t_{a} \beta_{a}^{*}$, put

$$
X(w)=\sum_{a \leq N}\left(\operatorname{deg}\left(\beta_{a}^{*}\right)-2\right) t_{a} \partial_{a} w-2 c_{1}(V) ;
$$

here we arrange the basis $\left\{\beta_{a}\right\}$ so that $\beta_{a}$ is an even class if and only if $a \leq N$. We say that $V$ is semi-simple in the sense of Dubrovin, if for a generic $w$, the quantum multiplication $X(w) \bullet_{w}$ on $H^{*}(V, \mathbb{C})$ has only simple eigenvalues. The question we are interested in is the following conjecture $[\mathrm{T}]$ :

Conjecture.(Tian) Any Fano manifold is semi-simple in the sense of Dubrovin.

The conjecture can be checked for complex projective spaces and quadratic hypersurfaces in projective spaces (cf. [KM], [T]).

There is a weaker version of the above conjecture. Let $H_{i n v}^{*}(V, \mathbb{C})$ be the subring of $H^{*}(V, \mathbb{C})$ with the cup product, generated by $H^{2}(V, \mathbb{C})$. For any algebraic variety $V$, it is believed (cf. [T]) that for any $w \in H_{i n v}^{*}(V, \mathbb{C})$, the quantum multiplication $\bullet_{w}$ preserves the subspace $H_{i n v}^{*}(V, \mathbb{C})$, namely, if $\alpha, \beta$ are in $H_{i n v}^{*}(V, \mathbb{C})$, so is $\alpha \bullet w$. Let us define $X_{i n v}(w)$ be the restriction of $X$ to $H_{i n v}^{*}(V, \mathbb{C})$. Assuming that the above belief is true, then $X_{i n v}$ acts on $H_{i n v}^{*}(V, \mathbb{C})$, and we can expect that $X(w) \bullet_{w}$ is semi-simple for a generic $w$ in $H_{i n v}^{*}(V, \mathbb{C})$, whenever $V$ is Fano (cf. [T]).

Now, let $V \subset \mathbf{P}^{n+r}$ be a Fano complete intersection of dimension $n \geq 3$. Then the ordinary cohomology algebra $H^{*}(V, \mathbb{C})$ is generated by the hyperplane class $H$ and the primitive cohomology $H^{n}(V, \mathbb{C})_{o}$. In particular, $H_{\text {inv }}^{*}(V, \mathbb{C})$ is the subspace of $H^{*}(V, \mathbb{C})$ generated by the hyperplane class $H$. It was observed in $[\mathrm{T}]$ that for any $w \in H_{i n v}^{*}(V, \mathbb{C})$, the quantum multiplication $\bullet_{w}$ preserves the subspace $H_{i n v}^{*}(V, \mathbb{C})$. Then $X_{i n v}$ acts on $H_{i n v}^{*}(V, \mathbb{C})$ for any $w$ in $H_{i n v}^{*}(V, \mathbb{C})$. An important case of the above conjecture is to prove the semi-simplicity of $X(w) \bullet_{w}$ for any complete intersection $V$ and a generic $w$ in $H_{i n v}^{*}(V, \mathbb{C})$. The result we have is the following:

Theorem 1. Let $V \subset \mathbf{P}^{n+r}(n \geq 3)$ be a smooth complete intersection of degree $\left(d_{1}, d_{2}, \cdots, d_{r}\right)$. If $n>2 \sum_{i=1}^{r}\left(d_{i}-1\right)-1\left(\right.$ except $n=7$ and $\left.\sum_{i=1}^{r}\left(d_{i}-1\right)=2\right)$, then $X(w) \bullet_{w}$, which acts on $H_{i n v}^{*}(V, \mathbb{C})$, is semi-simple in the sense of Dubrovin for a generic $w \in H_{i n v}^{*}(V, \mathbb{C})$.

Here we use Beauville's computation of quantum cohomology algebra for complete intersections satisfying the condition in the above theorem (cf. [B]). It seems that this condition on degree can be removed by using recent results in $[\mathrm{G}]$. 
Our motivation for the study of semi-simplicity comes from the following result of Dubrovin $[\mathrm{D}]$ : if $\left\{\alpha_{1}, \cdots, \alpha_{m}\right\}$ is a basis of $H^{*}(V, \mathbb{C})$ such that the quantum multiplication $X(w) \bullet_{w}$ on $H^{*}(V, \mathbb{C})$ with respect to this basis has only simple eigenvalues, then the integrable system defined by using the GromovWitten prepotential $\Phi^{V}$ (see section 2) can be extented meromorphically to $\left(\mathbf{P}^{1}\right)^{m}$.

The main tool of this paper is an elementary lemma in section 1 , the rest are standard computations. The method should also apply to some other Fano complete intersections. In this note, by a rational curve on $V$ we mean a simple genus 0 J-holomorphic curve for some generic almost complex structure $J$ on $V$.

Throughout this paper we work over the complex number field $\mathbb{C}$.

\section{An algebraic lemma}

The main tool of our computations is the following elementary lemma.

Lemma 1. Assume that

$$
g(y, z)=y^{m}+g_{1}(z) y^{m-1}+\cdots+g_{m}(z)
$$

is a polynomial of $y$, and $g_{1}(z), \cdots, g_{m}(z)$ are holomorphic functions of $z=$ $\left(z_{1}, z_{2}, \cdots, z_{N}\right)$ defined in a neighborhood of $0=(0, \cdots, 0) \in \mathbb{C}^{N}$. If the only repeated root of the polynomial $g(y, 0)=0$ is $y=0$, and the polynomial $g(y, z)=$ 0 does not have distinct roots for generic $z \in \mathbb{C}^{N}$, then

$$
g_{m}(z)=0 \quad \bmod \left(z_{1}, z_{2}, \cdots, z_{N}\right)^{2}
$$

that is, the constant term and linear terms of $g_{m}(z)$ are all 0.

Proof. Let $\mathcal{O}_{0}$ denote the ring of holomorphic functions defined in some neighborhood of $(0,0) \in \mathbb{C}^{N} \times \mathbb{C}$. Since all the repeated roots of $g(y, z)=0$ appear nearby $y=0$, we will study $g(y, z)$ in $\mathcal{O}_{0}$. By $[\mathrm{GH}]$ (page $8-11$ ), $\mathcal{O}_{0}$ is a unique factorization domain module units, we can write

$$
g(y, z)=\left(f_{1}(y, z)\right)^{i_{1}} \cdots\left(f_{h}(y, z)\right)^{i_{h}}
$$

where $f_{i}(y, z) \in \mathcal{O}_{0}$ are distinct and irreducible Weierstrass polynomials in $y$.

Now we claim that $i_{j}>1$ for some $j$. Otherwise, let $V_{j}=\left\{f_{j}(y, z)=0\right\}$, then

$$
g(y, z)=f_{1}(y, z) \cdots f_{h}(y, z), \quad\{g(y, z)=0\}=V_{1} \cup \cdots \cup V_{h} .
$$

Since the Weierstrass polynomial $f_{i}(y, z)$ is irreducible, and $\frac{\partial f_{i}}{\partial y}(y, z)$ has degree lower than $f_{i}(y, z)$ in $y$, we conclude that $f_{i}(y, z)$ and $\frac{\partial f_{i}}{\partial y}(y, z)$ are relative prime in $\mathcal{O}_{0}$. Hence the analytic variety

$$
\left\{f_{i}(y, z)=0\right\} \cap\left\{\frac{\partial f_{i}}{\partial y}(y, z)=0\right\} \subset \mathbb{C}^{N} \times \mathbb{C}
$$


has dimension $N-1<\operatorname{dim} \mathbb{C}^{N}$. Therefore the polynomial $f_{i}(y, z)=0$ has distinct roots for generic $z \in \mathbb{C}^{N}$.

Similarly, the analytic variety $\left\{f_{i}=0\right\} \cap\left\{f_{j}=0\right\} \subset \mathbb{C}^{N} \times \mathbb{C}$ has dimension $N-1<N$ for $i \neq j$ by Weak Nullstellensatz ([GH] p. 11). Hence the polynomials

$$
f_{i}(y, z)=0, \quad f_{j}(y, z)=0
$$

do not have common roots for generic $z \in \mathbb{C}^{N}$. This contradicts our assumption that $g(y, z)=0$ has repeated roots for generic $z$.

We conclude from above that $i_{j}>1$ for some $j$. Now $f_{j}(y, z)$ is not a unit in $\mathcal{O}_{0}$, we have $f_{j}(0,0)=0$, so $f_{j}(0, z) \in\left(z_{1}, \cdots, z_{N}\right)$, that is,

$$
g(0, z)=g_{m}(z)=f_{1}^{i_{1}} \cdots f_{h}^{i_{h}}(0, z) \in\left(z_{1}, \cdots, z_{N}\right)^{2}
$$

because $i_{j}>1$. So the lemma is proved.

\section{The computations}

We now start our computations. For simplicity of notations, we will assume that $q=\left(q_{1}, \cdots, q_{s}\right)=q_{1}=1$ in the rest of the paper.

Let $V \subset \mathbf{P}^{n+r}$ be a smooth complete intersection of degree $\left(d_{1}, d_{2}, \cdots, d_{r}\right)$, and

$$
\beta_{a}=H^{n+1-a} \in H_{2(a-1)}(V, \mathbb{C}), \quad 1 \leq a \leq n+1
$$

the $(n+1-a)$-th power of the hyperplane $H$, then $\left\{\beta_{a}\right\}$ is a basis for the homology group $H_{\text {inv }}(V, \mathbb{C})$. Any cohomology class $w \in H_{\text {inv }}^{*}(V, \mathbb{C})$ can be written as

$$
w=t_{1} \beta_{1}^{*}+t_{2} \beta_{2}^{*}+\cdots+t_{n+1} \beta_{n+1}^{*},
$$

here $\beta_{a}^{*}=H^{n+1-a} \in H^{2(n+1-a)}(V, \mathbb{C})(1 \leq a \leq n+1)$ in the ordinary cohomology. Then

$$
\begin{gathered}
\Phi_{i n v}^{V}\left(t_{1}, \cdots, t_{n+1}\right)= \\
\frac{d}{6} \sum_{a+b+c=2 n+3} t_{a} t_{b} t_{c}+\sum_{k \geq 1} \sum_{\left\{k_{a}\right\} \in S_{V, k}} \frac{\sigma_{k}^{V}\left(k_{1}, \cdots, k_{n-1}\right) t_{1}^{k_{1}} \cdots t_{n-1}^{k_{n-1}}}{k_{1} ! \cdots k_{n-1} !} e^{k t_{n}}, \\
S_{V, k}=\left\{\left\{k_{a}\right\}_{1 \leq a \leq n-1} \mid \sum_{i=1}^{n-1} i k_{n-i}=\left(n+r+1-\sum_{i=1}^{r} d_{i}\right) k+n-3>0\right\}, \\
\left(\beta_{a}^{*} \bullet \beta_{b}^{*}\right)\left(\beta_{c}\right)=\frac{\partial^{3} \Phi^{V}}{\partial t_{a} \partial t_{b} \partial t_{c}}(w),
\end{gathered}
$$

where $d=d_{1} d_{2} \cdots d_{r}$ is the degree of $V, \Phi^{V}$ is the Gromov-Witten prepotential, and $\sigma_{k}^{V}\left(k_{1}, \cdots, k_{n-1}\right)$ is the number of degree $k$ rational curves in $V$ through $k_{1}$ points, $\cdots, k_{n-1}$ subspaces of dimension $n-2$ in general position. 
Proposition 2, 3 in $[\mathrm{B}]$ and its higher degree analogy imply that there is a small positive number $\varepsilon=\varepsilon\left(d_{1}, \cdots, d_{r}, n\right)>0$, such that $\Phi_{i n v}^{V}\left(t_{1}, \cdots, t_{n+1}\right)$ is well-defined and holomorphic in $t_{1}, \cdots, t_{n+1}$ when $\left|t_{1}\right|<\varepsilon, \cdots,\left|t_{n+1}\right|<\varepsilon$ (also cf. $[\mathrm{G}],[\mathrm{J}],[\mathrm{T}])$.

Since $X(w) \bullet_{w}$ is a linear operator on $H_{i n v}^{*}(V, \mathbb{C})$, its matrix $A\left(t_{1}, \cdots, t_{n+1}\right)$ with respect to the basis $H^{0}=1, H^{1}, \cdots, H^{n}$ of $H_{i n v}^{*}(V, \mathbb{C})$ in the ordinary cohomology is a $(n+1) \times(n+1)$ matrix. Denote

$$
\begin{aligned}
G\left(\lambda, t_{1}, \cdots, t_{n+1}\right) & =\operatorname{det}\left(\lambda I-A\left(t_{1}, \cdots, t_{n+1}\right)\right), \\
e & =1+\sum_{i=1}^{r}\left(d_{i}-1\right) .
\end{aligned}
$$

In $[\mathrm{B}],[\mathrm{J}],[\mathrm{G}],[\mathrm{CJ}]$, it was proved that

$$
G(\lambda, 0, \cdots, 0)=\lambda^{n+1}-d_{1}^{d_{1}} \cdots d_{r}^{d_{r}} \lambda^{e-1},
$$

when $e<n+1$ and $n \geq 3$. Therefore, we have

Lemma 2. The only repeated root of the polynomial $G(\lambda, 0, \cdots, 0)=0$ is $\lambda=0$ when $e<n+1$ and $n \geq 3$.

Moreover, it is easy to see that $G(\lambda, 0, \cdots, 0)$ has distinct roots when $e=1,2$ (cf. $[\mathrm{KM}],[\mathrm{T}]$ ), that is, $X(w) \bullet_{w}$ is semi-simple when $e=1,2$ with $w=0$. Hence we may assume that $e \geq 3$.

We choose

$$
w=t_{2 e-3} \beta_{2 e-3}^{*}=t_{2 e-3} H^{n-2 e+4} \in H_{i n v}^{*}(V, \mathbb{C})
$$

when $n>2 e-3$, and assume that $t_{2 e-3}$ is very close to 0 . The rest of the computation will be made $\bmod \left(t_{2 e-3}\right)^{2}$.

Consider

$$
(n-j) k_{j}+(n-m) k_{m}+(n-2 e+3) k_{2 e-3}=(n+2-e) k+(n-3) .
$$

If $k_{2 e-3}=0$, then we have $k=1$ and $j+m=e+1$ because of the assumption $n>2 e-3$. If $k_{2 e-3}=1$, then we have either $k=1$ and $j+m=n-e+4$, or $k=2$ and $j+m=2$.

Hence the $(n+1) \times(n+1)$-matrix for $H^{1} \bullet w$ with respect to the basis $H^{0}=$ $1, H^{1}, \cdots, H^{n}$ of $H_{i n v}^{*}(V, \mathbb{C})$ in the ordinary cohomology is

$$
\left(\begin{array}{ccccccccc}
0 & 1 & 0 & \ldots & 0 & \ldots & 0 & \ldots & 0 \\
0 & 0 & 1 & \ldots & 0 & \ldots & 0 & \ldots & 0 \\
\vdots & \vdots & \vdots & \ddots & \vdots & \ddots & \vdots & \ddots & 0 \\
a_{1} t_{2 e-3} & 0 & 0 & \ldots & 1 & \ldots & 0 & \ldots & 0 \\
0 & a_{2} t_{2 e-3} & 0 & \ldots & 0 & \ldots & 0 & \ldots & 0 \\
\vdots & \vdots & \vdots & & \vdots & & \vdots & \ddots & 0 \\
b_{1} & 0 & 0 & \ldots & 0 & \ldots & 1 & \ldots & 0 \\
0 & b_{2} & 0 & \ldots & 0 & \ldots & 0 & \ldots & 0 \\
\vdots & \vdots & \vdots & & \vdots & & \vdots & \ddots & 1 \\
2 c_{1} t_{2 e-3} & 0 & 0 & \ldots & b_{e} & \ldots & a_{n-e+3} t_{2 e-3} & \ldots & 0
\end{array}\right)
$$


Here $e<n-e+3 \leq n$ because of the assumption $n>2 e-3$ and $e \geq 3$. When $e=3$, we have $a_{1}=a_{n-e+3}=a_{n}=0$ as $k_{j}$ is only defined for $1 \leq j \leq n-1$.

We next compute the matrix for $H^{n-2 e+4} \bullet_{w}$. We only need to do the computation $\bmod \left(t_{2 e-3}\right)$ here.

Consider

$$
(n-2 e+3) k_{2 e-3}+(n-j) k_{j}+(n-m) k_{m}=(n+2-e) k+(n-3) .
$$

We choose $k_{2 e-3}=1$ here. We have either $k=1$ and $j+m=n-e+4$, or $k=2$ and $j+m=2$. Hence the matrix for $H^{n-2 e+4} \bullet_{w}$ is

$$
\left(\begin{array}{ccccccccc}
0 & 0 & \ldots & 1 & 0 & \ldots & 0 & \ldots & 0 \\
0 & 0 & \ldots & 0 & 1 & \ldots & 0 & \ldots & 0 \\
\vdots & \vdots & \ddots & \vdots & \vdots & \ddots & \vdots & \ddots & \vdots \\
a_{1} & 0 & \ldots & 0 & 0 & \ldots & 1 & \ldots & 0 \\
0 & a_{2} & \ldots & 0 & 0 & \ldots & 0 & \ldots & 0 \\
\vdots & \vdots & & \vdots & \vdots & & \vdots & \ddots & \vdots \\
c_{1} & 0 & \ldots & 0 & 0 & \ldots & a_{n-e+3} & \ldots & 0
\end{array}\right)
$$

Here the 1 in the first row appears in the $(n-2 e+5)$-th column.

Now

$$
\begin{aligned}
X(w) & =(n-2 e+3) t_{2 e-3} H^{n-2 e+4}-(n-e+2) H^{1}, \\
-\frac{1}{n-e+2} X(w) & =H^{1}-\delta t_{2 e-3} H^{n-2 e+4}
\end{aligned}
$$

here $\delta=\frac{n-2 e+3}{n-e+2}$, and $0<\delta<1$ because $n>2 e-3$ and $e \geq 3$.

Therefore the matrix for $\left(H^{1}-\delta t_{2 e-3} H^{n-2 e+4}\right) \bullet_{w}$ with respect to the basis $H^{0}=1, H^{1}, \cdots, H^{n}$ of $H_{i n v}^{*}(V, \mathbb{C})$ in the ordinary cohomology is

$$
A(t)=\left(\begin{array}{cccccccccccc}
0 & 1 & 0 & \ldots & -t & 0 & \ldots & 0 & \ldots & 0 & \ldots & 0 \\
0 & 0 & 1 & \ldots & 0 & -t & \ldots & 0 & \ldots & 0 & \ldots & 0 \\
\vdots & \vdots & \vdots & & \vdots & \vdots & & \vdots & & \vdots & \ddots & \vdots \\
a_{1}^{*} t & 0 & 0 & \ldots & 0 & 0 & \ldots & 1 & \ldots & -t & \ldots & 0 \\
0 & a_{2}^{*} t & 0 & \ldots & 0 & 0 & \ldots & 0 & \ldots & 0 & \ldots & 0 \\
\vdots & \vdots & \vdots & & \vdots & \vdots & & \vdots & & \vdots & \ddots & \vdots \\
b_{1} & 0 & 0 & \ldots & 0 & 0 & \ldots & 0 & \ldots & 1 & \ldots & 0 \\
0 & b_{2} & 0 & \ldots & 0 & 0 & \ldots & 0 & \ldots & 0 & \ldots & 0 \\
\vdots & \vdots & \vdots & & \vdots & \vdots & & \vdots & & \vdots & \ddots & 1 \\
c_{1}^{*} t & 0 & 0 & \ldots & 0 & 0 & \ldots & b_{e} & \ldots & a_{n-e+3}^{*} & \ldots & 0
\end{array}\right)
$$

here $t=\delta t_{2 e-3}, a_{i}^{*}=\left(\delta^{-1}-1\right) a_{i}$, and $c_{1}^{*}=\left(2 \delta^{-1}-1\right) c_{1}$. 
Now let $A_{i}(t)$ be the matrix obtained from $A(t)$ by replacing the $i$-th column of $A(t)$ by its derivative with respect to $t$. A detailed computation shows that

$$
\begin{aligned}
\operatorname{det} A_{1}(0) & =(-1)^{n}\left(c_{1}^{*}-a_{1}^{*} b_{e}\right), \\
\operatorname{det} A_{n-e+3}(0) & =(-1)^{n+1} b_{1}\left(a_{n-e+3}^{*}+b_{e}\right), \\
\operatorname{det} A_{j}(0) & =0 \quad \text { for } j \neq 1, n-e+3 .
\end{aligned}
$$

As a result,

$$
\begin{aligned}
\operatorname{det} A(t) & =\left(\operatorname{det} A_{1}(0)+\operatorname{det} A_{2}(0)+\cdots+\operatorname{det} A_{n+1}(0)\right) t \\
& =(-1)^{n}\left(c_{1}^{*}-a_{1}^{*} b_{e}-a_{n-e+3}^{*} b_{1}-b_{1} b_{e}\right) t \quad \bmod \left(t^{2}\right) .
\end{aligned}
$$

Following Beauville [B] we use $d l_{j}$ to denote the number of lines in $V$ meeting two general linear space of codimension $n-j$ and $n+1-e+j$ respectively. We know that the varieties of lines and conics (with respect to the complex structure of $V$ ) contained in $V$ have the expected dimensions when $V$ is general [B].

In the case $e>3$, we have $b_{1}=b_{e}=l_{0}$. By proposition 2 in [B], we have

$$
a_{1}=a_{n-e+3}=l_{0} .
$$

By the corollary of proposition 3 in $[\mathrm{B}]$, we have $c_{1}=\frac{1}{2} l_{0}^{2}$. Therefore

$$
\begin{aligned}
c_{1}^{*}-a_{1}^{*} b_{e}-a_{n-e+3}^{*} b_{1}-b_{1} b_{e} & =c_{1}^{*}-2 a_{1}^{*} b_{1}-b_{1}^{2} \\
& =-\left(\delta^{-1}-\frac{1}{2}\right) l_{0}^{2} \\
& <0,
\end{aligned}
$$

because $0<\delta<1$. As a result, $\operatorname{det} A(t) \neq 0 \bmod \left(t^{2}\right)$.

In case $e=3, a_{1}=a_{n-e+3}=a_{n}=0$, we have

$$
\begin{aligned}
c_{1}^{*}-a_{1}^{*} b_{e}-a_{n-e+3}^{*} b_{1}-b_{1} b_{e} & =\left(\delta^{-1}-\frac{3}{2}\right) l_{0}^{2} \\
& =\left(\frac{n-1}{n-3}-\frac{3}{2}\right) l_{0}^{2} \\
& \neq 0
\end{aligned}
$$

except $n=7$ and $e=3$. Again, we have $\operatorname{det} A(t) \neq 0 \bmod \left(t^{2}\right)$.

Finally, we give

Proof of Theorem 1. By the above computation, we have

$$
\begin{aligned}
G\left(0, \cdots, 0, t_{2 e-3}, 0, \cdots, 0\right) & =\operatorname{det}\left(-A\left(0, \cdots, 0, t_{2 e-3}, 0, \cdots, 0\right)\right) \\
& =\operatorname{det}(-A(t)) \\
& \neq 0 \quad \bmod \left(t_{2 e-3}^{2}\right) .
\end{aligned}
$$

Therefore, Lemma 1 and 2 implies that the polynomial $G\left(\lambda, 0, \cdots, 0, t_{2 e-3}, 0\right.$, $\cdots, 0)=0$ has distinct roots for generic $t_{2 e-3} \in \mathbb{C}$. However, being semi-simple is an open conditions, we conclude that the polynomial

$$
G\left(\lambda, t_{1}, \cdots, t_{n+1}\right)=0
$$

has distinct roots for generic $\left(t_{1}, \cdots, t_{n+1}\right) \in \mathbb{C}^{n+1}$, that is, $X(w) \bullet_{w}$ is semisimple for generic $w$. 


\section{References}

[B] A. Beauville, Quantum cohomology of complete intersections, alg-geom/9501008.

[CJ] A. Collino and M. Jinzenji, On the structure of small quantum cohomology rings for projective hypersurfaces, preprint (1996).

[D] B. Dubrovin, Integrable systems in topological field theory, Nucl. Phys. B379 (1992), 627-689; also in Geometry of 2d topological field theory, preprint (1994).

[G] A. Givental, Equivariant Gromov-Witten invariants, preprint (1996).

[GH] P. Griffiths and J. Harris, Principles of Algebraic Geometry (1978), Wiley, New York.

$[\mathrm{J}] \quad$ M. Jinzenji, On quantum cohomology rings for hypersurfaces in $\mathbb{C} P_{N-1}$, preprint, hep-th/9511206.

[KM] M. Kontsevich and Yu. Manin, Gromov-Witten classes, quantum cohomology, and enumerative geometry, Comm. Math. Phys. 164 (1994), 525-562.

[MS] D. McDuff and D. Salamon, J-holomorphic curves and quantum cohomology, Amer. Math. Soc., University Lectures Series, 6, 1994.

[RT] Y. Ruan and G. Tian, A mathematical theory of quantum cohomology, J. Diff. Geom. 42 (1995), 259-367.

[T] G. Tian, Quantum cohomology and its associativity, Proc. of 1st Current developments in Mathematics, Cambridge (1995).

Department of Mathematics, M. I. T., Cambridge, MA 02139

E-mail address: tian@math.mit.edu

Department of Mathematics, Johns Hopkins University, Baltimore, MD 21218

E-mail address: geng@math.jhu.edu 